\title{
Disease Progression in Cutaneous Squamous Cell Carcinoma Patients With Satellitosis and In-transit Metastasis
}

\author{
TIMOTHY D. SMILE ${ }^{1}$, DAVID X. XIONG ${ }^{2,3}$, VAMSI VARRA $^{2,4}$, IAN W. WINTER $^{1}$, BRANDON T. BEAL $^{5}$, \\ BRIAN R. GASTMAN ${ }^{6}$, JESSICA L. GEIGER ${ }^{7}$, DAVID J. ADELSTEIN ${ }^{7}$, WILMA F. BERGFELD $^{8}$, \\ MELISSA P. PILIANG ${ }^{8}$, STEVEN D. BILLINGS $^{8}$, JENNIFER S. KO $^{8}$, THOMAS J. KNACKSTEDT ${ }^{9}$, \\ JENNIFER L. LUCAS ${ }^{6}$, CHRISTINA M. POBLETE-LOPEZ ${ }^{6}$, JON G. MEINE ${ }^{6}$, \\ ALOK VIJ ${ }^{6}$, ALLISON T. VIDIMOS ${ }^{6}$ and SHLOMO A. KOYFMAN ${ }^{1}$ \\ ${ }^{1}$ Department of Radiation Oncology, Taussig Cancer Institute, Cleveland Clinic, Cleveland, OH, U.S.A.; \\ ${ }^{2}$ College of Medicine, Case Western Reserve University, Cleveland, OH, U.S.A.; \\ ${ }^{3}$ Department of Internal Medicine, University of Pittsburgh Medical Center, Pittsburgh, PA, U.S.A.; \\ ${ }^{4}$ Department of Internal Medicine, University Hospitals Medical Center, Cleveland, OH, U.S.A.; \\ ${ }^{5}$ Jacksonville Skin Cancer Specialists, Jacksonville, FL, U.S.A.; \\ ${ }^{6}$ Dermatology and Plastic Surgery Institute, Cleveland Clinic, Cleveland, OH, U.S.A.; \\ ${ }^{7}$ Department of Hematology and Medical Oncology, Taussig Cancer Institute, \\ Cleveland Clinic, Cleveland, OH, U.S.A.; \\ ${ }^{8}$ Institute of Pathology, Cleveland Clinic, Cleveland, OH, U.S.A.; \\ ${ }^{9}$ Department of Dermatology, MetroHealth Hospital, Cleveland, OH, U.S.A.
}

\begin{abstract}
Background/Aim: Satellitosis/in-transit metastasis ( $S$ ITM) has prognostic value in melanoma and Merkel cell carcinoma, but is not incorporated into cutaneous squamous cell carcinoma (cSCC) staging. Patients and Methods: From our IRB-approved registry, patients with high-risk cSCC, including patients with S-ITM, were identified. Univariate (UVA) and multivariate (MVA) analyses were performed to compare disease progression $(D P)$ and overall survival (OS). Cumulative incidence of DP and OS analyses were performed using FineGray and Kaplan-Meier methods, respectively. Results: A total of 18 S-ITM subjects were compared to 247 high risk subjects including T3NO (n=143), N1-N3 without extranodal extension (ENE) (n=56), N1-N3 with ENE $(n=26)$ and M1 disease $(n=22)$. Median follow up was 16.5 months. Three-year rates of DP were $22 \%$ for T3NO, 42\% for S-ITM, $48 \%$ for T4 bone invasion, $50 \%$ for N1-N3 without extranodal extension (ENE), 53\% for N1-N3 with ENE, and $66 \%$ for M1. Patients with S-ITM did not experience significantly worse DP compared to those with T3NO
\end{abstract}

This article is freely accessible online.

Correspondence to: Timothy D. Smile, MD, Cleveland Clinic, Department of Radiation Oncology, Taussig Cancer Institute, 9500 Euclid Avenue/CA-50, Cleveland, OH 44195, U.S.A. Tel: +1 2169041533, Fax: +1 2164451068, e-mail: smilet@ccf.org

Key Words: cSCC, satellitosis, in-transit metastasis.
$(H R=1.96,95 \% C I=0.8-4.9 ; p=0.14)$. Conclusion: Cutaneous SCC patients with S-ITM experienced outcomes similar to locally advanced non-metastatic cSCC patients. Larger studies are needed to guide incorporation into staging systems.

Cutaneous squamous cell carcinoma (cSCC) is common, with over 700,000 invasive cases reported in the U.S. annually (1). The majority of these cases are low risk with excellent prognosis after resection via Mohs microsurgery (MMS) or wide local excision (WLE) $(2,3)$. However, a small subset of cSCC patients have tumor risk factors that translate to increased disease recurrence, metastasis, and cause-specific mortality $(1,4)$. These risk factors include tumor size $>2 \mathrm{~cm}$, poorly-differentiated histology, perineural invasion (PNI) of a nerve $>0.1 \mathrm{~mm}$ in diameter, tumor invasion beyond fat, and erosion or invasion of the underlying bone. These results led to changes in the American Joint Committee on Cancer (AJCC) 8th edition staging system in 2016 (5) as well as to the development of a novel Brigham and Women's Hospital (BWH) staging system (6), both of which risk-stratify patients to help guide adjuvant therapy and follow up.

Satellitosis and in-transit metastasis (S-ITM) represent a rare but significant risk factor caused by intralymphatic metastases from cutaneous malignancies. S-ITM is known to purport worse outcomes in cutaneous melanoma and Merkel cell carcinoma (7-9). These data led to incorporation of SITM into the AJCC staging systems for cutaneous melanoma and Merkel cell carcinoma $(5,10-12)$. However, there is a 
relative paucity of data to understand the clinical significance of S-ITM in cSCC patients, thus far limited to small retrospective studies and several case reports (13-15). However, these data show that recurrence, metastasis and survival may be worse for cSCC patients with S-ITM.

S-ITM is not included in either the AJCC $8^{\text {th }}$ edition or BWH staging system. As such, these patients are not currently being categorized with regard to prognosis by the current staging system. As a result, clinicians face challenges regarding choice of therapy when a patient presents with SITM. The purpose of this study was to report outcomes for cSCC patients with S-ITM in order to provide prognostic context in comparison to other known risk factors.

\section{Patients and Methods}

Cohort. We queried an IRB-approved institutional database of patients with AJCC 8th edition stage III and IV cSCC who were treated surgically between 2010 and 2019. We included patients for whom surgical pathology samples were available for review. From this registry, patients who had S-ITM at the time of diagnosis defined by presence of dermal lesions that were clinically distinct from the primary tumor and located between the primary tumor and the first echelon lymphatics - were identified. Patients with tumor deposits located within a first echelon lymph node basin positive for nodal metastases were described as lymph node metastasis with extranodal extension (ENE) rather than S-ITM. Additionally, patients with high risk tumor characteristics were included for comparison to S-ITM patients. These cohorts included patients with T3N0 tumors, T4 tumors with bone invasion, N1 to N3 disease without ENE, N1 to N3 disease with ENE, and patients with M1 disease.

Endpoints. Endpoints included incidence of disease progression (DP) as a measure of success or failure of curative therapy. Disease progression was defined as any local, regional or distant failure with pathologic confirmation via biopsy or resection. We also analyzed the overall survival (OS) endpoint for each of the disease cohorts described above.

Statistics. Univariate (UVA) and multivariate (MVA) analyses were performed to determine risk factors associated with the primary outcome of DP. UVA and MVA analyses were also performed to determine OS and to compare S-ITM outcomes with other risk group cohorts. Cumulative incidence estimates for DP were performed using Fine-Gray method, and OS estimates were performed using the Kaplan-Meier method. Maximum likelihood estimates of the Fine-Gray cumulative incidence functions for DP were compared to T3N0 tumors using the pairwise hazard ratio method. Logrank comparisons of the survival functions of each cSCC cohort were estimated using the Chi-Square method.

\section{Results}

We identified 247 patients with cSCC with disease features including T3N0 ( $n=143)$, N1-N3 without ENE ( $n=56)$, N1N3 with ENE $(n=26)$, M1 disease $(n=22)$ and S-ITM $(n=18)$. The demographic patient and tumor characteristics are demonstrated in Table I. Chi-square comparisons showed no significant differences between groups regarding age, gender, smoking status, or immunosuppression status. There were significant differences between groups regarding histologic differentiation, specifically with T3N0 patients having a significantly higher proportion of well-differentiated tumors and M1 patients having a high proportion of unknown differentiation status $(p<0.0001)$. Of note, there were zero well-differentiated tumors among the 26 patients demonstrating nodal disease with ENE. A lower proportion of T3N0 patients had recurrent tumors (24\%) compared to the total cohort $(44 \%)$, a difference that was statistically significant $(p<0.0001)$. Presence of any perineural involvement was not significantly different between groups, although patients with M1 disease or those demonstrating nodal disease with ENE had numerically higher incidence of PNI with a $p$-value approaching significance $(p=0.06)$. FineGray cumulative incidence functions of disease progression were performed for each of the cohorts, as seen in Figure 1. Maximum likelihood estimates of the DP cumulative incidence estimates were compared to patients with T3N0 tumors. Patients with S-ITM did not have worse disease progression outcomes compared to T3N0 in this maximum likelihood estimate analysis $(p=0.14)$, though the other risk groups were each significantly more likely to have disease progression compared to $\mathrm{T} 3 \mathrm{NO}$, as shown in Table II. Product-limit survival estimates for each of the groups are demonstrated in Figure 2. Log-rank comparisons of the survival functions were compared between each cohort as shown in Table III. Of note, S-ITM patients had improved survival outcomes compared to M1 patients, but not compared to any of the other cohorts.

\section{Discussion}

Our study found that patients with S-ITM had a comparably high rate of disease progression relative to other locally advanced cSCC patients, but not as high as patients with M1 disease. S-ITM was associated with similar disease progression to patients with T3N0 tumors with improved outcomes compared with T4N0 patients without S-ITM. However, recurrence outcomes appear worse than those of the T3N0 cohort with a $p$-value approaching significance $(p=0.14)$ in an analysis limited by a small number of S-ITM patients. Patients with S-ITM were still curable, but we believe this risk factor has implications for guiding prognosis and adjuvant therapy. Patients with in-transit metastasis among other risk factors were observed to be curative in the TROG 05.01 phase III prospective randomized trial investigating whether the addition of concurrent chemotherapy to adjuvant radiotherapy improves locoregional control for high-risk cSCC patients (16). The investigators included S-ITM as a high-risk criterion for inclusion in the 


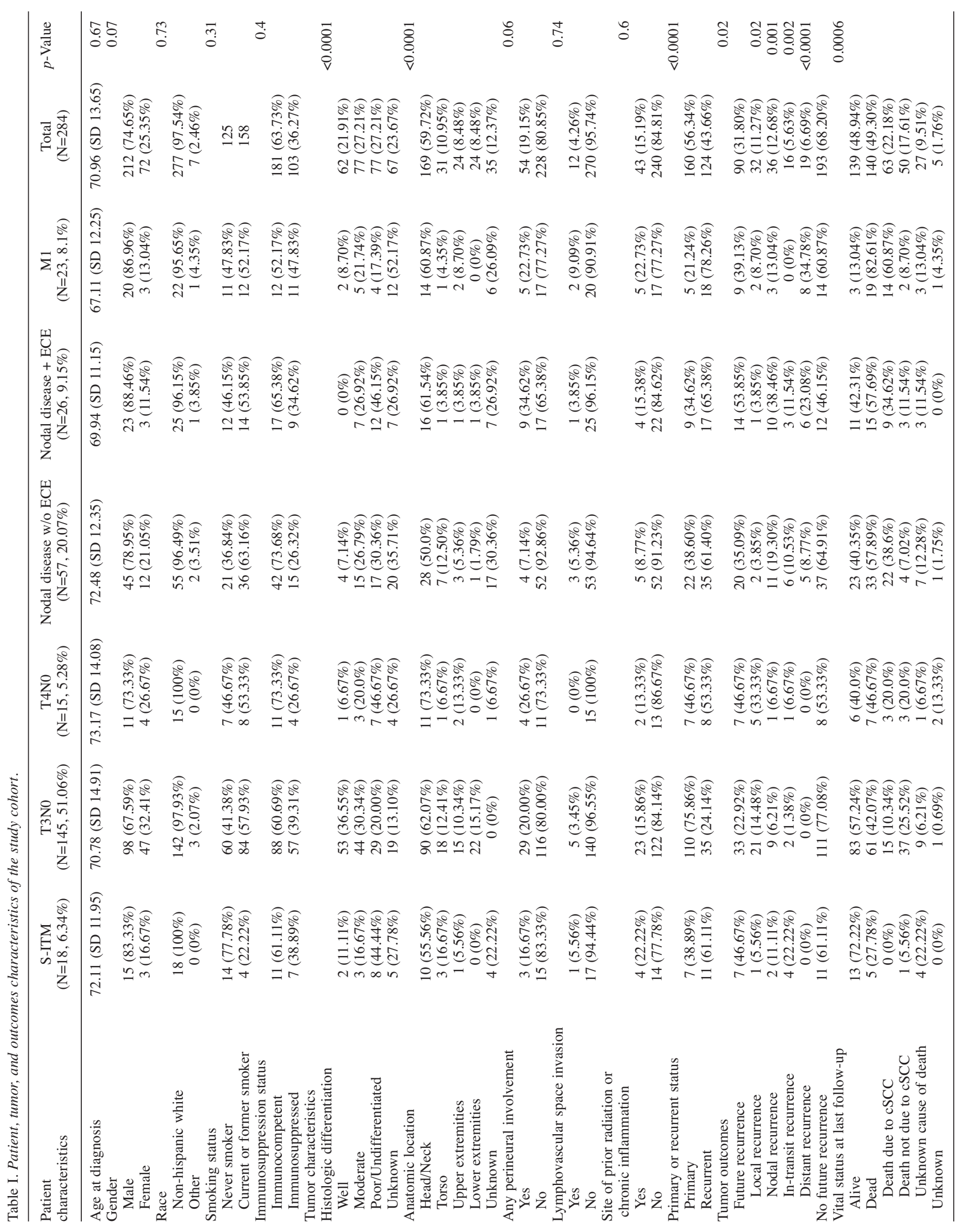




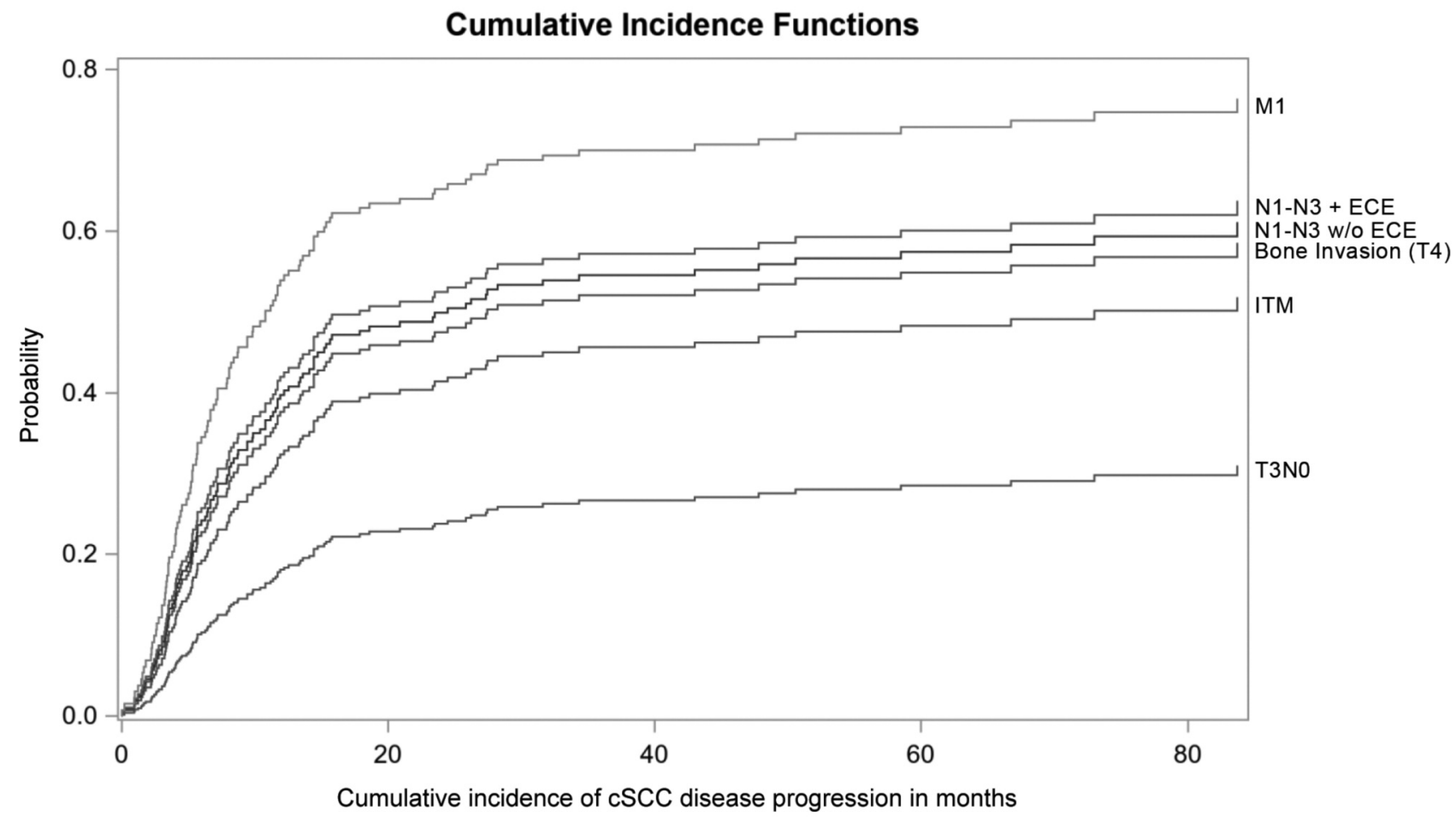

Figure 1. Fine-Gray Cumulative Incidence Functions (CIFs) of cSCC disease progression (defined as any recurrence or death due to SCC). S-ITM: Satellitosis/In-transit metastases; T3NO: cSCCs fulfilling the AJCC8 Criteria for T3 tumors (of note, these include tumors outside of the head and neck); N1-N3 w/o ECE: Tumors with any nodal spread without extra-capsular extension; N1-N3 + ECE: Tumors with any nodal spread with extracapsular extension; $M 1$ : distantly metastatic tumors.

trial, ultimately enrolling 56 patients in total with S-ITM found on surgical pathology. While this trial demonstrated excellent outcomes with adjuvant RT with no observed benefit to the addition of chemotherapy, analyses regarding the prognostic importance of S-ITM were not performed. As such, we believe our analysis reveals that S-ITM has important implications for cSCC staging.

Previous multicenter case series have examined the effect of S-ITM on survival outcomes for patients with cSCC. In the series of 31 patients from Australia and New Zealand by Ma et al., S-ITM was associated with overall survival rates of $27 \%$ at 3 years and $13 \%$ at 5 years (14). Median follow up for the study was 12 months. Most tumors (94\%) occurred in the head and neck area, and the majority of patients $(68 \%)$ were immunocompetent. Another multicenter case series by Carucci et al. examined survival outcomes for cSCC patients with S-ITM, many of whom were immunosuppressed because of history of organ transplantation (15). The authors reviewed 21 patients with in-transit metastases, of whom 15 were organ transplant recipients. With a mean follow up of 24 months, only $33 \%$ of organ transplant patients had no evidence of disease. In contrast, $80 \%$ of the immunocompetent cohort was without evidence of disease. In organ transplant patients, 33\%
Table II. Maximum likelihood estimates of the Fine-Gray cumulative incidence functions for cSCC disease progression compared to T3NO tumors.

\begin{tabular}{lrc}
\hline Risk group & $p$-Value & Hazard ratio \\
\hline N1-N3 w/o ECE & 0.0002 & $2.54(95 \% \mathrm{CI}=1.55-4.17)$ \\
N1-N3 + ECE & 0.0008 & $2.73(95 \% \mathrm{CI}=1.52-4.90)$ \\
M1 & $<0.0001$ & $3.88(95 \% \mathrm{CI}=2.24-6.74)$ \\
S-ITM & 0.1448 & $1.96(95 \% \mathrm{CI}=0.79-4.86)$ \\
Bone invasion (T4) & 0.0173 & $2.37(95 \% \mathrm{CI}=1.16-4.82)$ \\
\hline
\end{tabular}

ECE: Extracapsular extension; S-ITM: satellitosis/in-transit metastasis; CI: confidence interval.

had died from disease and $33 \%$ were alive with either nodal or distant metastases. Because immunosuppression is an established risk factor for inferior survival among cSCC patients (17), the degree to which outcomes in this series were attributable to S-ITM versus the immunosuppressed status is unclear. This question was addressed by a recent series by $\mathrm{Xu}$ et al. who examined prognostic factors not included in AJCC 8th edition and their relationship to recurrence and survival in $101 \mathrm{cSCC}$ patients (18). While 


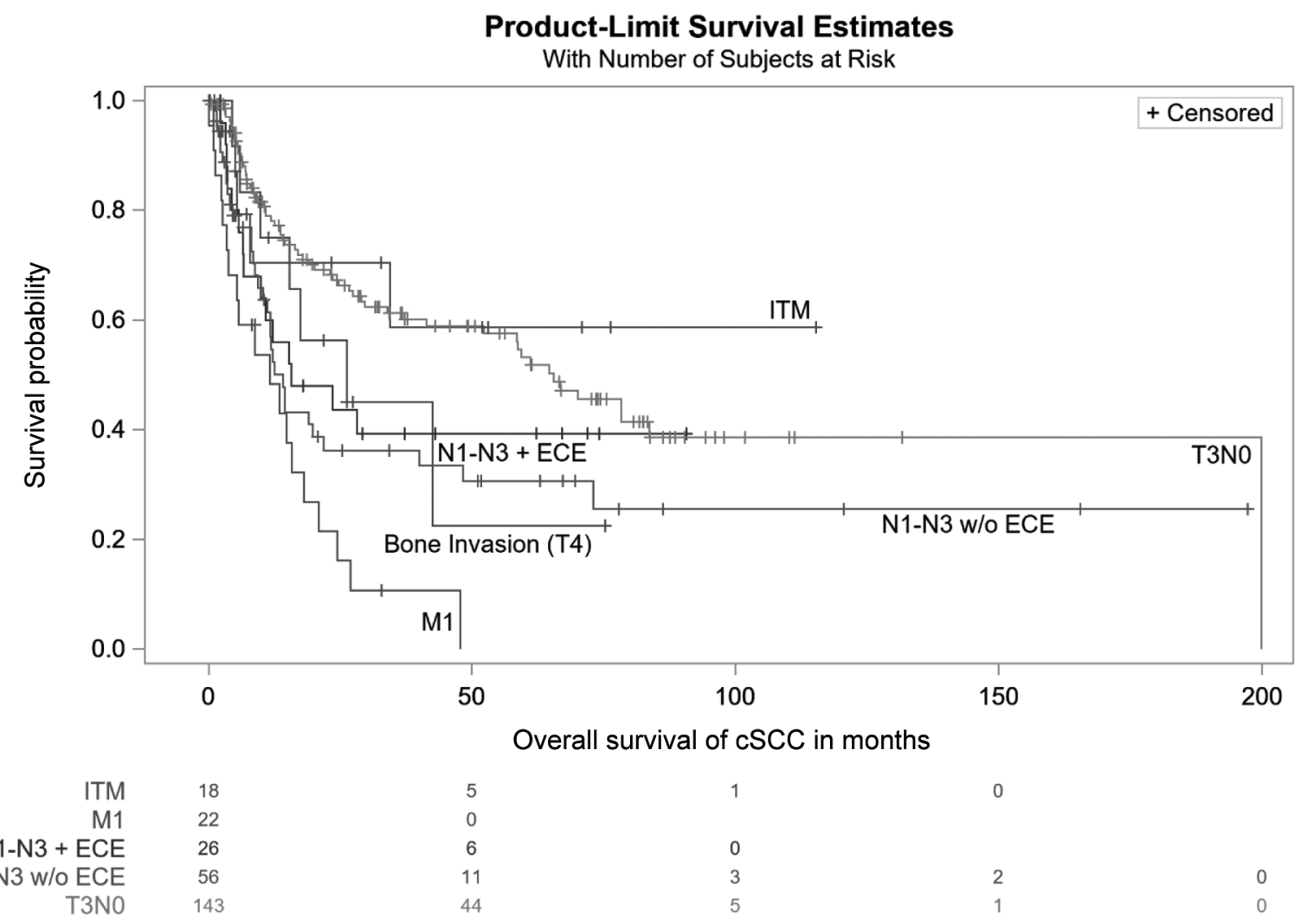

Figure 2. Kaplan-Meier overall survival functions for cSCC tumor groups. Groups are defined in the same way as above for the Fine-Gray CIFs.

limited by a small number of S-ITM patients $(n=7)$, the authors reported locoregional recurrence hazard ratios of 2.7 (1.3-5.7, $p=0.009)$ for immunosuppression and $9.1(2.4-35.1$, $p=0.001)$ for presence of S-ITM on multivariate analysis. The authors also reported that S-ITM was significantly associated with worse overall survival compared to patients without $(p=0.004)$.

In the context of these small prior retrospective studies, our study further demonstrates the need for additional study of S-ITM. A larger cohort of patients is needed to clarify the patterns of failure and survival outcomes for cSCC patients with S-ITM. Recurrence and survival outcomes may help stratify treatment decisions including potential intensification of adjuvant therapy with a risk of increased toxicity. On the contrary, perhaps immunocompetent patients with S-ITM should be stratified to initiate anti-PD1 immunotherapy rather than aggressive local therapy given the recent encouraging outcomes in phase II prospective data (18). Once a deeper understanding of the prognostic significance of S-ITM is achieved, we are advocating for incorporation of S-ITM into future staging systems. This will allow clinicians to document S-ITM and further promote the understanding of outcomes related to this risk factor.

\section{Conclusion}

Satellitosis and in-transit metastasis represents an aggressive form of cutaneous squamous cell carcinoma that is associated with trends toward worse outcomes. Patients with this risk factor are still curable with aggressive local therapy. Further study with larger cohorts of patients is required to clarify the importance of this risk factor for recurrence and survival.

\section{Conflicts of Interest}

Smile: none, Xiong: none, Varra: none, Winter: none, Beal: none, Gastman: none, Geiger: Research support to institution from Regeneron, Genentech, Alkermes; advisory board member for Regeneron, Adelstein: none, Bergfeld: none, Piliang: none, Billings: none, Ko: none, Knackstedt: none, Lucas: none, Poblete-Lopez: none, Meine: none, Vij: none, Vidimos: none, Koyfman: Consulting and research support for Merck, Research support for Bristol Meyers Squibb, Honoraria from UpToDate.

\section{Authors' Contributions}

Conception and design: Smile, Xiong, Koyfman; Administrative support: Smile, Vidimos, Koyfman; Provision of study materials or patients: Beal, Gastman, Geiger, Adelstein, Bergfeld, Piliang, 
Table III. Log-rank comparisons of the survival functions of each cSCC group.

\begin{tabular}{llcc}
\hline \multicolumn{2}{l}{ Adjustment for multiple comparisons for the log-rank test } & \\
\cline { 1 - 2 } Strata comparison & & \multirow{2}{*}{ Chi-Square } & $p$-Value \\
\cline { 1 - 2 } Group & Group & & \\
\hline \multirow{2}{*}{ Bone invasion (T4) } & S-ITM & & \\
Bone invasion (T4) & M1 & 0.9295 & 0.99 \\
Bone invasion (T4) & N1-N3 + ECE & 0.4625 & $\mathbf{0 . 0 3 1}$ \\
Bone invasion (T4) & N1-N3 w/o ECE & 3.067 & 1 \\
Bone invasion (T4) & T3N0 & 11.2541 & 0.71 \\
S-ITM & M1 & 14.8282 & $\mathbf{0 . 0 1 1 9}$ \\
S-ITM & N1-N3 + ECE & 1.2093 & 0.9914 \\
S-ITM & N1-N3 w/o ECE & 5.5634 & 0.2424 \\
S-ITM & T3N0 & 7.4236 & 0.0923 \\
M1 & N1-N3 + ECE & 4.9203 & 0.332 \\
M1 & N1-N3 w/o ECE & 0.1491 & 1 \\
M1 & T3N0 & 24.1788 & $<\mathbf{0 . 0 0 0 1}$ \\
N1-N3 + ECE & N1-N3 w/o ECE & 1.7151 & 0.9579 \\
N1-N3 + ECE & T3N0 & 9.7704 & $\mathbf{0 . 0 2 6 3}$ \\
N1-N3 w/o ECE & T3N0 & 12.8548 & $\mathbf{0 . 0 0 5}$ \\
\hline
\end{tabular}

S-ITM: Satellitosis/in-transit metastasis; ECE: extracapsular extension; T3N0: cSCC fulfilling the AJCC8 criteria for T3 tumors without nodal spread; N1-N3 w/o ECE: tumors with any nodal spread without extracapsular extension; N1-N3 + ECE: tumors with any nodal spread with extra-capsular extension; M1: distantly metastatic tumors. Significant $p$-Values are shown in bold.

Billings, Ko, Knackstedt, Lucas, Poblete-Lopez, Meine, Vij, Vidimos, Koyfman; Collection and assembly of data: Smile, Xiong, Varra, Winter; Data analysis and interpretation: Smile, Xiong; Manuscript writing: Smile, Winter, Koyfman; Final approval of manuscript: Smile, Xiong, Varra, Winter, Beal, Gastman, Geiger, Adelstein, Bergfeld, Piliang, Billings, Ko, Knackstedt, Lucas, Poblete-Lopez, Meine, Vij, Vidimos, Koyfman.

\section{Acknowledgements}

Supported by the Melvin Markey Discovery Fund at the Cleveland Clinic, OH, U.S.A.

\section{References}

1 Karia PS, Han J and Schmults CD: Cutaneous squamous cell carcinoma: estimated incidence of disease, nodal metastasis, and deaths from disease in the United States, 2012. J Am Acad Dermatol 68(6): 957-966, 2013. PMID: 23375456. DOI: 10.1016/j.jaad.2012.11.037

2 Alam M and Ratner D: Cutaneous squamous-cell carcinoma. New Engl J Med 344(13): 975-983, 2001. PMID: 6709010. DOI: 10.1056/NEJM198405033101805

3 Jambusaria-Pahlajani A, Miller CJ, Quon H, Smith N, Klein RQ and Schmults CD: Surgical monotherapy versus surgery plus adjuvant radiotherapy in high-risk cutaneous squamous cell carcinoma: a systematic review of outcomes. Dermatol Surg 35(4): 574-585, 2009. PMID: 19415791. DOI: 10.1111/j.15244725.2009.01095.x

4 Jambusaria-Pahlajani A, Kanetsky PA, Karia PS, Hwang W-T, Gelfand JM, Whalen FM, Elenitsas R, Xu X and Schmults CD: Evaluation of AJCC tumor staging for cutaneous squamous cell carcinoma and a proposed alternative tumor staging system. JAMA Dermatol 149(4): 402-410, 2013. PMID: 23325457. DOI: 10.1001/jamadermatol.2013.2456

5 Amin MB, Edge S, Greene F, Byrd DR, Brookland RK, Washington MK, Gershenwald JE, Compton CC, Hess KR, Sullivan DC, Jessup JM, Brierley JD, Gaspar LE, Schilsky RL, Balch CM, Winchester DP, Asare EA, Madera M, Gress DM, Meyer LR (eds.). AJCC Cancer Staging Manual. Springer, 2017.

6 Karia PS, Jambusaria-Pahlajani A, Harrington DP, Murphy GF, Qureshi AA and Schmults CD: Evaluation of American Joint Committee on Cancer, International Union Against Cancer, and Brigham and Women's Hospital tumor staging for cutaneous squamous cell carcinoma. J Clin Oncol 32(4): 327-334, 2014. PMID: 24366933. DOI: 10.1200/JCO.2012.48.5326

7 Read RL, Haydu L, Saw RP, Quinn MJ, Shannon K, Spillane AJ, Stretch JR, Scolyer RA and Thompson JF: In-transit melanoma metastases: incidence, prognosis, and the role of lymphadenectomy. Ann Surg Oncol 22(2): 475-481, 2015. PMID: 25256128. DOI: 10.1245/s10434-014-4100-0

8 Hayes AJ, Clark MA, Harries M and Thomas JM: Management of in-transit metastases from cutaneous malignant melanoma. $\mathrm{Br}$ J Surg 91(6): 673-682, 2004. PMID: 15164434. DOI: 10.1002/ bjs. 4610

9 Harms KL, Healy MA, Nghiem P, Sober AJ, Johnson TM, Bichakjian CK and Wong SL: Analysis of prognostic factors from 9387 Merkel cell carcinoma cases forms the basis for the New 8th edition AJCC Staging System. Ann Surg Oncol 23(11): 35643571, 2016. PMID: 27198511. DOI: 10.1245/s10434-016-5266-4

10 Buzaid AC, Ross MI, Balch CM, Soong S, McCarthy WH, Tinoco L, Mansfield P, Lee JE, Bedikian A, Eton O, Plager C, Papadopoulos N, Legha SS and Benjamin RS: Critical analysis of the current American Joint Committee on Cancer staging system for cutaneous melanoma and proposal of a new staging system. J Clin Oncol 15(3): 1039-1051, 1997. PMID: 9060544. DOI: 10.1200/JCO.1997.15.3.1039

11 Balch CM, Gershenwald JE, Soong SJ, Thompson JF, Atkins MB, Byrd DR, Buzaid AC, Cochran AJ, Coit DG, Ding S, Eggermont AM, Flaherty KT, Gimotty PA, Kirkwood JM, McMasters KM, Mihm MC Jr., Morton DL, Ross MI, Sober AJ and Sondak VK: Final version of 2009 AJCC melanoma staging and classification. J Clin Oncol 27(36): 6199-6206, 2009. PMID: 19917835. DOI: $10.1200 / J C O .2009 .23 .4799$

12 Gershenwald JE, Scolyer RA, Hess KR, Sondak VK, Long GV, Ross MI, Lazar AJ, Faries MB, Kirkwood JM, McArthur GA, Haydu LE, Eggermont AMM, Flaherty KT, Balch CM and Thompson JF; for members of the American Joint Committee on Cancer Melanoma Expert Panel and the International Melanoma Database and Discovery Platform: Melanoma staging: Evidencebased changes in the American Joint Committee on Cancer eighth edition cancer staging manual. CA Cancer J Clin 67(6): 472-492, 2017. PMID: 29028110. DOI: 10.3322/caac.21409

13 Xu MJ, Lazar AA, Garsa AA, Arron ST, Ryan WR, El-Sayed IH, George JR, Algazi AP, Heaton CM, Ha PK and Yom SS: Major prognostic factors for recurrence and survival independent of the 
American Joint Committee on Cancer eighth edition staging system in patients with cutaneous squamous cell carcinoma treated with multimodality therapy. Head Neck 40(7): 14061414, 2018. PMID: 29524273. DOI: 10.1002/hed.25114

14 Ma JH, Wu A, Veness M, Estall V, Hong A, Borg M, James C, Ibbetson J, Ooi C, Weightman W, McColl I, Hamann I, Grieve N, Ozluer S, Salmon P, Nikitins M, Caplash Y, Marshall N, Edwards T, Patterson I, Selva D and Huilgol SC: In-transit metastasis from squamous cell carcinoma. Dermatol Sug 42(11): 1285-1292, 2016. PMID: 27598442. DOI: 10.1097/DSS.000000 0000000864

15 Carucci JA, Martinez JC, Zeitouni NC, Christenson L, Coldiron $\mathrm{B}$, Zweibel S and Otley CC: In-transit metastasis from primary cutaneous squamous cell carcinoma in organ transplant recipients and nonimmunosuppressed patients: clinical characteristics, management, and outcome in a series of 21 patients. Dermatol Surg 30(4 Pt 2): 651-655, 2004. PMID: 15061850. DOI: 10.1111/j.1524-4725.2004.30151.x

16 Porceddu SV, Bressel M, Poulsen MG, Stoneley A, Veness MJ, Kenny LM, Wratten C, Corry J, Cooper S, Fogarty GB, Collins M, Collins MK, Macann AMJ, Milross CG, Penniment MG, Liu HY, King MT, Panizza BJ and Rischin D: Postoperative concurrent chemoradiotherapy versus postoperative radiotherapy in high-risk cutaneous squamous cell carcinoma of the head and neck: the randomized phase III TROG 05.01 trial. J Clin Oncol 36(13): 1275-1283, 2018. PMID: 29537906. DOI: 10.1200/ JCO.2017.77.0941
17 Manyam BV, Garsa AA, Chin RI, Reddy CA, Gastman B, Thorstad W, Yom SS, Nussenbaum B, Wang SJ, Vidimos AT and Koyfman SA: A multi-institutional comparison of outcomes of immunosuppressed and immunocompetent patients treated with surgery and radiation therapy for cutaneous squamous cell carcinoma of the head and neck. Cancer 123(11): 2054-2060, 2017. PMID: 28171708. DOI: 10.1002/cncr.30601

18 Migden MR, Rischin D, Schmults CD, Guminski A, Hauschild A, Lewis KD, Chung CH, Hernandez-Aya L, Lim AM, Chang ALS, Rabinowits G, Thai AA, Dunn LA, Hughes BGM, Khushalani NI, Modi B, Schadendorf D, Gao B, Seebach F, Li S, Li J, Mathias M, Booth J, Mohan K, Stankevich E, Babiker HM, Brana I, GilMartin M, Homsi J, Johnson ML, Moreno V, Niu J, Owonikoko TK, Papadopoulos KP, Yancopoulos GD, Lowy I and Fury MG: PD-1 blockade with cemiplimab in advanced cutaneous squamous-cell carcinoma. N Engl J Med 379(4): 341-351, 2018. PMID: 29863979. DOI: 10.1056/NEJMoa1805131

Received September 17, 2020

Revised November 27, 2020

Accepted November 28, 2020 